\section{La extensión universitaria argentina desde la promoción y evaluación estatal}

Mariano Damián Negro

Universidad Nacional de Entre Ríos y

Universidad Autónoma de Entre Ríos,

Argentina.

marianodnegro@gmail.com

\section{Julieta Clara Gómez}

Instituto Universitario CEMIC (Centro

de Educación Médica e Investigaciones

Científicas) y Universidad de Buenos

Aires, Argentina.

julietaclarago@gmail.com
A 100 años de la Reforma Universitaria de 1918 /

Perspectivas

RECEPCIÓN: 29/06/17

ACEPTACIÓN FINAL: 24/09/17

\section{Resumen}

Si bien desde la Reforma Universitaria (1918) se considera a la extensión universitaria como una función central de la universidad junto a la docencia y la investigación, en la práctica se la desarrolló en menor medida.

Con la sanción de la Ley de Educación Superior (LES) y la creación de la Comisión Nacional de Evaluación y Acreditación Universitaria (CONEAU), el sistema universitario argentino experimentó un cambio en materia de evaluación estatal. Desde su creación, la Comisión realizó más de 120 evaluaciones externas con recomendaciones de mejora sobre las distintas funciones. Nos propusimos identificar el lugar que ocupa la extensión analizando 29 de ellas.

Se encontró que las recomendaciones referidas a la extensión son menores que las efectuadas a otras funciones universitarias y que ocupan un lugar relegado dentro de la jerarquía universitaria. Esta situación se acentúa en las instituciones privadas, mientras que las universidades públicas han impulsado la extensión gracias a partidas presupuestarias específicas promovidas en la última década. Es decir que, en lo que refiere a la formulación de políticas públicas para el fomento de la extensión universitaria en nuestro país, en la última década se observó un importante crecimiento en lo que respecta al financiamiento de proyectos y programas que la promuevan.

\section{Palabras clave}

- Extensión universitaria

- Evaluación institucional

- Promoción de la extensión

- CONEAU

- Recomendaciones

\section{Resumo}

Embora seja considerada a função de extensão universitária central da universidade com o ensino e pesquisa na prática da Reforma Universitária (1918) de extensão desenvolvido em menor grau.

Com a promulgação da Lei do Ensino Superior e da criação de CONEAU (avaliação e agência de acreditação), o sistema universitário argentino sofreu uma mudança na avaliação do estado.

Desde a sua criação, a CONEAU fez mais de 120 avaliações externas com recomendações para a melhoria nas diferentes funções. Propusemo-nos a identificar o local da extensão analisando 29 deles.

Verificou-se que as recomendações relacionadas com a extensão são mais baixos do que aqueles feitos para outras funções universitárias e ocupando um lugar relegado dentro da hierarquia universitária; Isto é acentuado em instituições privadas, enquanto as universidades públicas têm desenvolvido de extensão, graças a rubricas orçamentais específicas promovidas na última década. Ou seja, quando se trata da formulação de políticas públicas para a promoção da extensão universitária em nosso país na última década um crescimento significativo em relação ao financiamento de projectos e programas que promovam foi observada.

\section{Palavras-chave}

- Extensão universitária

- Avaliação institucional

- Promover a extensão

- CONEAU

- Recomendações
Para citación de este artículo

Negro, M. D. y Gómez, J. C. (2017). La extensión

universitaria argentina desde la promoción y

evaluación estatal. Revista $+E$ versión en línea, 7(7), 46-59. Santa Fe, Argentina: Ediciones UNL. 


\section{Introducción}

Si bien la Reforma Universitaria de 1918 marcó un punto de inflexión en lo que refiere al propósito y la función de la extensión universitaria al considerarla función central de la universidad junto a la docencia y la investigación, en la práctica la extensión se desarrolló en menor medida: en el caso de las universidades privadas, la extensión resulta incipiente cuando no inexistente, y en el caso de las universidades públicas, la función ha tomado mayor impulso a través de partidas presupuestarias específicas. Con la creación de la Comisión Nacional de Evaluación y Acreditación Universitaria (CONEAU) a partir de la sanción de la Ley de Educación Superior (LES) de 1995, el sistema universitario argentino experimentó un cambio con relación a la evaluación por parte del Estado sobre el cumplimiento de las funciones sustantivas.

A lo largo de 20 años, la CONEAU realizó 120 informes de evaluación institucional en los que estableció recomendaciones de mejora en cuanto a las distintas funciones universitarias: docencia, investigación y extensión.

Asimismo, en lo que refiere a la formulación de políticas públicas para el fomento de la extensión universitaria en nuestro país, en la última década se observó un importante crecimiento respecto de la financiación de proyectos y programas que la promuevan. Este trabajo, que se enmarca en una investigación más amplia, ${ }^{1}$ tiene por objeto comprender la situación actual de la extensión universitaria en cuanto a identificar el lugar que ocupa la extensión en las evaluaciones externas de CONEAU a partir del análisis de 29 informes de evaluación externa. A la vez, pretende identificar y describir las distintas políticas estatales que se suscitaron en el período 2005-2015 con la intención de promover la extensión en las instituciones de educación superior.

El artículo abordará, en primer lugar, un recorrido histórico sobre la extensión universitaria argentina; en segundo lugar, las múltiples definiciones que han dado las instituciones universitarias a la extensión; en tercer lugar, se describirá la política estatal de promoción de la extensión en las universidades públicas en el período 2005-2015; en cuarto lugar, se detallarán los aspectos metodológicos de la investigación; y en quinto lugar, se desarrollará el análisis que surge de la sistematización de las recomendaciones de los informes de evaluación externa estudiados. Para finalizar, se presentará un apartado con las conclusiones.

\section{La extensión universitaria y su desarrollo en Argentina}

El fenómeno de la extensión universitaria es mundial. Sus primeros antecedentes se encuentran en Europa y en Estados Unidos. Aproximadamente en el año 1870 surgió la extensión universitaria en Inglaterra con el objetivo de extender el saber que se generaba en la universidad al pueblo trabajador. Fueron los mismos trabajadores los que demandaron a la universidad otro tipo de conocimientos más acordes con las capacidades y habilidades que requería el avance industrial a través de la Cámara de los Lores. La respuesta no tardó en llegar: el profesor James Stuart, de la Universidad de Cambridge, preparó los primeros cursos para cumplir con este requerimiento y dio lugar a lo que luego pasó a denominarse university extention. Estos cursos tuvieron mucho éxito y se multiplicaron inmediatamente. Así, le siguieron la Universidad de Oxford (1878) con las lecturas de extensión del reverendo Arthur Johnson, al igual que otras instituciones inglesas. Esta forma de transmisión de conocimientos se propagó rápidamente por todo el continente europeo. La adoptaron varias universidades e instituciones con algunos matices que obedecían a sus realidades, como sucedió en Alemania, Austria, Bélgica, Francia, Hungría, Italia (Turín), Rusia y, posteriormente, España. Un caso especial fue Francia ya que en ella nacieron las universidades populares, por lo cual la extensión se circunscribió a las grandes conferencias y a las actividades culturales (Herrera Albrieu, 2012). En 1862, en Estados Unidos, a partir del Acta de Morril se promovieron acciones de formación y actividades con las comunidades rurales.

"Luego la Hatch Act de 1887, concedió los recursos necesarios para las investigaciones aplicadas y las acciones experimentales. Es decir que la iniciativa para que las instituciones de educación superior se relacionen con su comunidad, proviene desde 1862, lo cual constituye un antecedente de la extensión universitaria en Inglaterra". (Herrera Albrieu, 2012:2)

En América Latina, el primer antecedente se produjo en Argentina, particularmente en la Universidad Nacional de La Plata en el año 1905, al incorporar a la extensión en el estatuto de la Universidad. Sin embargo, como se señaló, la Reforma Universitaria de 1918 marcó un momento bisagra en el sistema universitario argentino al considerar a la extensión como una función central en el vidad de académica y de investigación desarrollada por los autores en la Universidad Nacional de Entre Ríos y en el Instituto Universitario CEMIC. 
quehacer de las universidades. Desde entonces, se consideró a la extensión como una función social inherente e inseparable de la universidad que se reflejaba en los postulados reformistas como la necesidad de abrir la universidad al pueblo en tanto deber social. Esto significó poner el saber universitario al servicio de la sociedad y hacer de sus problemas un aspecto importante de su ocupación. De esta manera, se introdujo en nuestro país la idea de que las universidades son instituciones en las cuales se preserva, se crea y se promueve la cultura (Isa, 2012).

En 1920, el Centro de Estudiantes de la Facultad de Derecho de la Universidad de Buenos Aires inauguró sus "Cursos de extensión universitaria" con esta indicación:

"Juzgamos, por lo tanto, perentoria la conveniencia de devolver a la colectividad siquiera alguna parte de los beneficios que una situación de fortuna nos permite extraer del patrimonio espiritual de la especie, reclamando al propio tiempo, la igualación de las oportunidades, para que cada individuo pueda adquirir toda la porción proporcionada a sus aptitudes intelectuales. No le basta, sin embargo, a la generación de la paz, criticar defectos del pasado. Quiere practicar el descontento activo. Quiere ser una energía creadora. Quiere construir. Ya que el pueblo no va hacia la universidad, ésta, representada por sus órganos positivos, irá hacia el pueblo, procurando llenar su verdadera misión de docencia social”. (Cúneo, 2010:25)

Para ese entonces, ya existían experiencias extensionistas en varias universidades latinoamericanas y la extensión se consolidaba como uno de los elementos centrales del ideario y el programa del movimiento de la Reforma (Carlevaro, 1986).

Hacia mediados del siglo pasado esta función experimentó un cambio ya que la extensión en Argentina desarrolló "una tendencia recurrente a asumir actividades de educación popular de adultos, desarrollada por jóvenes intelectuales, militantes políticos, sociales o religiosos" (Argumedo, 2002:15).

Luego de la década de los '70, el modelo extensionista de la universidad argentina tomó un tinte orientado hacia la satisfacción de la demanda del mercado. Este corrimiento significó

"desatender la originaria relación universidad-sociedad para enfocar en otra expresada en la ecuación universidad-mercado mediada por las condiciones propuestas por el paradigma de la eficiencia y la eficacia" (Pozo, 2012:2).

Ya en los años 90 se apuntó a reducir la práctica de la extensión en Argentina a un

"conjunto de actividades conducentes a organizar los recursos disponibles, identificar los problemas y demandas del medio, coordinar las correspondientes acciones de transferencia, reorientar y recrear las actividades de docencia e investigación a partir de la interacción con el medio". (Argumedo et al., 2002:17)
En este contexto también se dio una notable expansión del sistema universitario que potenció no solo la diversidad de acciones sino también las denominaciones de "extensión". Varias universidades optaron por otras denominaciones debido a la supuesta carga "transferencista" que contenía el nombre. Superado el modelo extensionista enmarcado en el neoliberalismo argentino, se produjo un nuevo viraje. En la última década se entiende que las políticas de extensión asumen la construcción de espacios destinados a generar interacciones entre la universidad y los variados actores sociales: se considera imprescindible que la universidad asuma su compromiso real con la comunidad, que atienda demandas y trabaje con asociaciones intermedias, sectores de la producción, instituciones públicas y privadas y población en general. Se considera que la práctica de la extensión universitaria, además, contribuirá para formar profesionales con la mayor conciencia social, comprometidos en la solución de los problemas (Isa, 2012). A su vez, surge con más fuerza en este contexto la perspectiva antropológica que advierte que estas propuestas solo serán posibles en la medida en que se reconozca "que no se trataría de compartir una cultura única y excelsa, sino de establecer un vínculo intercultural comunicativo de un mayor valor fecundante, en pos de luchar por condiciones de mayor justicia" (4).

En síntesis, y siguiendo a Menoni (2014), la extensión (polisémica y heterogénea) es una función característica de las universidades latinoamericanas herederas del movimiento reformista de comienzos del siglo XX. Ni en la universidad colonial, ni en su heredera republicana inmediata (que adoptó el modelo "napoleónico" fuertemente profesionalista), ni en las formaciones universitarias de otras regiones, la extensión ha tenido un arraigo y un desarrollo tal como el que presenta en América Latina (Tünnermann, 2000). No obstante, en comparación con el despliegue de las funciones de docencia y de investigación, su desarrollo ha sido menor.

\section{La extensión universitaria objeto de múltiples definiciones} Como se ha señalado, desde su origen la extensión ha ocupado diferentes lugares en las universidades y es formulada y ensayada desde diversas perspectivas en relación con diferentes motivaciones. En términos generales, se indica que la extensión universitaria representa el vínculo existente entre universidadsociedad a través de sus diferentes formas de manifestación, facilitando además la prestación de servicios a la comunidad universitaria y a la población en general, para dar respuesta a las necesidades de superación y capacitación y contribuyendo al desarrollo cultural integral.

En el sistema universitario argentino existen diferentes nominaciones para las secretarías que cumplen la función bajo análisis. Si bien la mayoría lleva el nombre de extensión universitaria, existen instituciones que utilizan otras denominaciones: "Secretaría 
de Cooperación y Servicio Público", "Dirección de extensión y seguimiento de graduados", "Centro de Servicios y Centro Cultural", "Secretaría de Gestión Comunitaria". Ello no solo es una característica de las instituciones universitarias argentinas sino también de las latinoamericanas. Tal como lo expresa Gómez de Mantilla (2011), no hay univocidad con relación a la manera de denominar la función. Algunos la llaman simplemente extensión, proyección social e, incluso, proyección universitaria. Otros han privilegiado el concepto y la práctica de la divulgación cultural y algunas denominaciones hacen referencia a lo que se conoce como vinculación o la Responsabilidad Social Universitaria (RSU) respecto de diferentes sectores de la sociedad; y más recientemente en algunas universidades se la ha bautizado como "integración universitaria" (Herrera Albrieu, 2012).

Del análisis de los informes de evaluación externa elaborados por CONEAU surge que, en función de la heterogeneidad de las actividades realizadas y de las instituciones universitarias que integran el sistema universitario argentino, sumada a la ausencia de una definición consensuada a nivel nacional, en la actualidad la extensión universitaria continúe siendo conceptualizada de diferentes modos que podrían ser agrupados en categorías para facilitar su comprensión. Es de destacar que en la práctica estas categorías aparecen combinadas:

- Extensión entendida como actividad de extensión curricular: son aquellas actividades de extensión integradas a las curriculares de las carreras. Estas actividades formativas, a cargo de los docentes designados responsables de las asignaturas correspondientes mediante la capacitación, prevención, orientación, información o asesoramiento a la comunidad, permiten complementar la formación teórico-curricular de los estudiantes.

- Extensión entendida como actividades de extensión extracurricular: son aquellas actividades de extensión que se desarrollan fuera del horario curricular, con carácter optativo para alumnos, docentes e investigadores. Son promovidas por la institución y están orientadas a la comunidad universitaria en general, a la ciudadanía y a los distintos actores sociales. Habitualmente estas actividades generan espacios propicios para el desarrollo de la cultura y el deporte.

- Extensión entendida como actividades de transferencia: son aquellas actividades sistemáticas que transfieren a la sociedad los conocimientos y las experiencias producidas por la investigación. De esta manera, las acciones de extensión adquieren el carácter de transferencia de conocimientos disponibles y utilizables, en función de las demandas y requerimientos de los diversos actores sociales y económicos y permiten la formación permanente de la comunidad académica (docentes, investigadores, egresados) y de la comunidad científica en general.

- Extensión entendida como vinculación tecnológica: son aquellas acciones de transferencia tecnológica llevadas a cabo por unidades dependientes de las universidades que permiten acceder a diversas fuentes de financiamiento, tanto públicas como privadas, y que se desarrollan con el objeto de vincular principalmente a los sectores productivos de la región con las áreas científicas y tecnológicas universitarias.

- Extensión entendida como bienestar estudiantil: son aquellas actividades destinadas a alumnos y/o graduados de la universidad. Se refiere, por ejemplo, a aquellos programas de becas de estudio para los estudiantes que tienen como objetivo sostener su ingreso a la universidad, permanencia y egreso. Por lo general están orientadas a alumnos de bajos recursos que tienen dificultades económicas para ingresar y permanecer en la universidad o bien que, por razones específicas vinculadas a la disciplina y al mercado de trabajo, abandonan los estudios una vez alcanzadas ciertas competencias que les permiten insertarse laboralmente.

De los informes analizados surge también que las universidades argentinas han otorgado a la extensión diferentes funciones y actividades dentro de su estructura institucional. En algunos casos se incluye el bienestar universitario y el seguimiento de graduados; en un número menor se incorpora a la vinculación tecnológica; otras desarrollan la política comunicacional y se ocupan, a su vez, de la política editorial cuando la hubiera. La capacitación es el común denominador de todas las actividades y también de una manera generalizada lo son las actividades culturales.

\section{La promoción de la extensión desde el Estado}

En distintos trabajos (Espinoza e Isa, 2012; Pozo, 2012; Chiroleau, 2006; Herrera Albrieu, 2012) se indica que en los últimos diez años (2005-2015) el desarrollo de las actividades de extensión universitaria ha cobrado un fuerte impulso en la mayoría de las universidades nacionales. En lo que respecta a las políticas de extensión universitaria, estos cambios estuvieron asociados a un marcado incentivo de la actividad a partir del incremento de los fondos presupuestarios específicos, históricamente deficitarios con relación a las partidas presupuestarias asignadas a la investigación y la docencia (funciones centrales de las universidades de gestión pública).

Se mencionan a continuación algunos ejemplos de los programas estatales impulsados en la última década que permiten comprender concretamente por qué las universidades públicas han desarrollado la extensión en mayor magnitud en los últimos años; esto es, debido a la asignación de partidas presupuestarias específicas otorgadas por el Ministerio de Educación de la Nación y otros organismos nacionales.

La Dirección Nacional de Desarrollo Universitario y Voluntariado del Ministerio de Educación de la Nación comenzó a implementar, en el año 2006, el Programa de Voluntariado Universitario (PVU) 
Tabla 1: Programa de Voluntariado Universitario. Cantidad de proyectos y tipo de participación. 2009-2013

\begin{tabular}{|c|c|c|c|c|c|c|}
\hline $\begin{array}{l}\text { Proyectos de Voluntariados } \\
\text { aprobados }\end{array}$ & $\begin{array}{l}\text { Universidades } \\
\text { participantes }\end{array}$ & $\begin{array}{l}\text { Cantidad } \\
\text { de proyectos }\end{array}$ & $\begin{array}{l}\text { Organizaciones } \\
\text { civiles participantes }\end{array}$ & $\begin{array}{l}\text { Participación docente } \\
\text { e investigadores }\end{array}$ & $\begin{array}{l}\text { Participación } \\
\text { estudiantil }\end{array}$ & Financiamiento \\
\hline Primera convocatoria 2009 & 40 & 424 & 1200 & 2200 & 10.800 & $\$ 8.810 .052$ \\
\hline Segunda convocatoria 2009 & 42 & 240 & 584 & 975 & 3657 & $\$ 4.399 .994$ \\
\hline $\begin{array}{l}\text { Convocatoria } 2010 \\
\text { del Bicentenario }\end{array}$ & 40 & 510 & 1050 & 2000 & 8781 & $\$ 9.971 .916$ \\
\hline $\begin{array}{l}\text { Convocatoria específica } \\
\text { "La Universidad se conecta } \\
\text { con la igualdad" } 2011\end{array}$ & 33 & 139 & 436 & 139 & 2487 & $\$ 2.986 .762$ \\
\hline Convocatoria anual 2011 & 47 & 588 & 1243 & 2585 & 10.637 & $\$ 12.488 .479$ \\
\hline Convocatoria anual 2012 & 47 & 646 & 3176 & 2779 & 28.192 & $\$ 13.999 .954$ \\
\hline $\begin{array}{l}\text { Convocatoria específica } \\
\text { "La Universidad se conecta } \\
\text { con la igualdad" } 2012\end{array}$ & 40 & 180 & 385 & 673 & 2369 & $\$ 3.992 .242$ \\
\hline Convocatoria anual 2013 & 47 & 684 & 1711 & 2439 & 9744 & $\$ 15.668 .386$ \\
\hline $\begin{array}{l}\text { Convocatoria Específica } \\
\text { "La Patria es el Otro" } 2013\end{array}$ & 4 & 61 & 137 & 673 & 2369 & $\$ 1.474 .125$ \\
\hline TOTAL & - & 3472 & - & - & - & $\$ 73.791 .910$ \\
\hline
\end{tabular}

Fuente: elaboración propia sobre la base de la información publicada por el Ministerio de Educación de la Nación, 2016.

Tabla 2: Programa Nacional de Becas. Presupuesto y cantidad de becas por tipo de Programa. Año 2013

\begin{tabular}{|c|c|c|}
\hline Programa & Cantidad de becas & Presupuesto \\
\hline PNBU & 16.669 & $\$ 60.008 .400$ \\
\hline PNBB & 28.644 & $\$ 213.816 .900$ \\
\hline PNBTIC & 16 & $\$ 192.000$ \\
\hline TOTAL & 45.329 & $\$ 274.017 .300$ \\
\hline
\end{tabular}

Fuente: elaboración propia sobre la base de la información publicada en el Anuario SPU, 2013, Ministerio de Educación de la Nación.
Tabla 3: Programa Universidad, Diseño y Desarrollo Productivo

\begin{tabular}{|c|c|c|c|}
\hline $\begin{array}{l}\text { Programa Universidad, Diseño } \\
\text { y Desarrollo Productivo }\end{array}$ & 2013 & 2014 & 2015 \\
\hline $\begin{array}{l}\text { Universidades e institutos } \\
\text { universitarios nacionales } \\
\text { participantes }\end{array}$ & 30 & 37 & 33 \\
\hline Proyectos financiados & 126 & 197 & 213 \\
\hline Financiamiento & $\$ 2.885 .871$ & $\$ 4.513 .128$ & $\$ 6.051 .095$ \\
\hline Docentes participantes & 597 & 792 & 704 \\
\hline Alumnos participantes & 909 & 1399 & 1426 \\
\hline Graduados Participantes & $\mathrm{s} / \mathrm{i}$ & $\mathrm{s} / \mathrm{i}$ & 110 \\
\hline
\end{tabular}

s/i: sin información.

Fuente: elaboración propia sobre la base de la información publicada por el Ministerio de Educación de la Nación, 2013-2015. 
con "el objetivo general de profundizar la función social de la Universidad, integrando el conocimiento generado en las aulas con las problemáticas más urgentes de nuestro país" (SPU, 2013). Entre los años 2009 y 2013 se presentó un total de 3472 proyectos de extensión universitaria a las diferentes convocatorias abiertas por el PVU, lo que representó un financiamiento total de \$73.791.910. Por otra parte, cabe destacar que hasta al año 2007 el único programa de otorgamiento de becas que funcionaba era el Programa Nacional de Becas Universitarias (PNBU). A partir de 2008, mediante la creación del Programa Nacional de Becas Bicentenario (PNBB) y el Programa Nacional de Becas de Grado TIC (PNBTIC), se diferencian los programas en función de ramas disciplinares: las PNBB están dirigidas a los estudiantes que cursan carreras científico-técnicas; las PNBTIC, orientadas a aquellos que cursan carreras de grado relacionadas con las tecnologías de la información y la comunicación, y las PNBU a todas las demás carreras. Tomaremos como referencia lo sucedido con este Programa durante el año 2013, en el que se financiaron 45.329 becas por un monto de $\$ 274.017 .300$. Ver Tabla 2.

De igual modo, en 2013, 2014 y 2015 la Dirección Nacional de Desarrollo Universitario y Voluntariado de la Secretaría de Políticas Universitarias del Ministerio de Educación de la Nación convocó a estudiantes, docentes e investigadores de carreras vinculadas con la ingeniería, el diseño, las ciencias aplicadas, la tecnología y otras afines, a la presentación de proyectos que se orientaran al desarrollo de productos innovadores que propusieran soluciones productivas (destinada a atender ciertas necesidades de la comunidad, mejorar procesos de producción o solucionar problemáticas concretas). Lo hizo a través del Programa Universidad, Diseño y Desarrollo Productivo (PUDyDP). Ver Tabla 3.

En 2013, la Secretaría de Políticas Universitarias impulsó el Programa "Universidad y Trabajo Argentino" con el objetivo de promover la vinculación entre las universidades y el sector productivo, de manera de instar en especial a la generación de proyectos relacionados con la asistencia a la exportación de productos. En la primera convocatoria se financiaron 63 proyectos por un total de $\$ 4.770 .014$, y en la convocatoria del año 2014 se aprobaron 61 proyectos por un monto total de $\$ 4.808 .642$.

Además, se conjugó al financiamiento específico a las instituciones universitarias un sistema de respaldo dirigido a jóvenes entre 18 y 24 años para terminar la escuela primaria, la secundaria, estudiar un oficio o carrera universitaria y que no se encontraran trabajando o percibieran un ingreso menor a tres salarios mínimo, vital y móvil y que su grupo familiar poseyera iguales condiciones. Con el objetivo de mejorar sus trayectorias educativas se lanzó en 2014 el Programa de Respaldo a Estudiantes de Argentina (Progresar), una acción conjunta entre distintos organismos del Estado: los Ministerios de Ciencia, Tecnología e Innovación Productiva; de Economía y Finanzas Públicas; de Trabajo, Empleo y Seguridad
Social; de Educación; de Desarrollo Social; de Salud; de Defensa; de Seguridad; del Interior y Transporte, y la Administración Nacional de la Seguridad Social (ANSES).

En el informe "Invertir para Transformar, la juventud como protagonista del desarrollo", la Comisión Económica para América Latina y el Caribe, CEPAL (2014), señaló que solo durante el primer año el Plan Progresar logró una reducción de 19 \% en el coeficiente Gini entre jóvenes de 15 y 29 años desde 2004 a 2014, y destacó el incremento en el gasto público medido en dólares por joven de $493 \%$ en el mismo período.

\section{La evaluación institucional}

La CONEAU ha institucionalizado las funciones que le corresponden legalmente de manera progresiva. Desde 1996, evalúa proyectos institucionales. A partir de 1997 evalúa informes anuales de instituciones universitarias con autorización provisoria, realiza evaluaciones externas y acredita posgrados. Desde 1999 evalúa solicitudes de reconocimiento definitivo y de agencias privadas de evaluación y acreditación de carreras de grado (CONEAU, 2012). Los antecedentes de estas funciones se pueden reconocer en la creación de la Secretaría de Políticas Universitarias del Ministerio de Educación en el año 1993, que firmó convenio con algunas universidades (de gestión pública y privada) para planificar y elaborar mecanismos en conjunto que ayuden a la evaluación institucional. Estos acuerdos ya preveían los procesos de autoevaluación a cargo de las mismas instituciones, que servirían de marco para las evaluaciones externas. Asimismo, se creó en el seno del entonces Ministerio de Educación y Cultura de la Nación, en el año 1994, la Comisión de Acreditación de Posgrados (CAP) que, antes de la sanción de la LES, realizó una convocatoria para la acreditación voluntaria de maestrías y doctorados que se venían dictando en universidades de gestión pública y privadas, a lo que respondieron 300 programas en total y significó la evaluación y acreditación de un número importante de carreras de posgrado (Marquis, 2014).

Una vez que comenzó a funcionar CONEAU luego de la sanción de la LES, el Ministerio de Educación le transfirió las funciones de evaluación de instituciones de educación superior y las desarrolladas por CAP, dando por cerradas las funciones de la Comisión de Acreditación de Posgrado. De este modo, en la LES se establece, en su artículo $46^{\circ}$, que la CONEAU es un organismo descentralizado que trabaja en jurisdicción del Ministerio de Educación de la Nación y tiene las siguientes funciones:

a) Coordinar y llevar adelante la evaluación externa prevista en el artículo $44^{\circ}$ de la Ley de Educación Superior.

b) Acreditar las carreras de grado a que se refiere el artículo $43^{\circ}$ de la Ley de Educación Superior, así como las carreras de posgrado, cualquiera sea el ámbito en que se desarrollen, conforme a los 
estándares que establezca el Ministerio de Educación en consulta con el Consejo de Universidades.

c) Pronunciarse sobre la consistencia y viabilidad del proyecto institucional que se requiere para que el Ministerio de Educación autorice la puesta en marcha de una nueva institución universitaria nacional con posterioridad a su creación o el reconocimiento de una institución universitaria provincial.

d) Preparar los informes requeridos para otorgar la autorización provisoria y el reconocimiento definitivo de las instituciones universitarias privadas, así como los informes en base a los cuales se evaluará el periodo de funcionamiento provisorio de dichas instituciones.

Determina que las instituciones universitarias tienen autonomía académica e institucional (artículo 29\%) pero así y todo deberán garantizar instancias de evaluación que tendrán por objeto analizar los logros y dificultades en el cumplimiento de sus funciones $y$ sugerir medidas para su mejoramiento (artículo $44^{\circ}$ ). Con relación a ello, en 1997 la CONEAU elaboró un documento: "Lineamientos para la evaluación institucional" (aprobado por Resolución CONEAU N 94/97) que expone la concepción general de la evaluación institucional, establece dos etapas (la autoevaluación y la evaluación externa), y define el marco normativo y las características de las evaluaciones externas que realiza.

"La evaluación institucional tiene como fin primordial el mejoramiento de la calidad de las instituciones que brindan formación universitaria. Asimismo, el respeto irrestricto de la autonomía universitaria pone en valor la singularidad y la heterogeneidad de los proyectos institucionales que desarrollan las instituciones universitarias. La evaluación institucional debe ser útil a la propia institución universitaria y a la comunidad que utiliza sus servicios en general”. (CONEAU, 2012:28)

Es decir que las evaluaciones institucionales permiten analizar los logros y dificultades en el cumplimiento de las funciones que tiene toda universidad (docencia, extensión e investigación). En el caso de las universidades nacionales, además, se evalúa la gestión. No obstante, para que la autoevaluación cumpla con el objetivo de la mejora institucional, la normativa que rige el procedimiento indica que resulta fundamental que la institución:

“a) cuente con un alto grado de participación de la comunidad universitaria;

b) adopte una perspectiva contextual e histórica referida a sus objetivos y al proyecto institucional;

c) aborde el conjunto de funciones que desempeña la institución;

d) enfoque a la institución como un todo entendiendo que su identidad es más que la suma de sus partes; y

e) que el informe autoevaluación brinde una imagen documentada de la institución" (CONEAU, 2012).
De este modo, como señalan Krotsch, Camou, y Prati (2007), se evidencian algunas de las razones que explican el carácter estratégico de los procesos de evaluación:

- La función de rendición de cuentas de la evaluación institucional: ante la demanda de diferentes sectores de la sociedad de una educación universitaria pertinente y de calidad, la evaluación de los procesos y resultados permite dar cuenta del modo en que se gastan los recursos públicos (en el caso de las universidades nacionales).

- La evaluación como información valiosa: el proceso le brinda a la propia universidad información sistemática sobre sí misma, de manera tal que el proceso de autoevaluación se convierte en una instancia de aprendizaje institucional, y el conocimiento obtenido, un valioso insumo para realizar las transformaciones necesarias para adecuarse a las nuevas demandas internas y externas (mejorando la toma de decisiones).

- La evaluación como herramienta de gestión: la evaluación permite obtener información diferencial de los procesos y los rendimientos, y asignar recursos en forma racional.

La combinación de estos aspectos constituye el uso sustantivo de la evaluación institucional en tanto sean incorporados de manera regular y estable a la cultura institucional. Este uso se diferencia del uso instrumental o formalista de la evaluación (Krotsch, Camou y Prati, 2007).

El proceso de evaluación institucional se inicia con la firma de un acuerdo entre la CONEAU y la institución universitaria que manifieste su interés por evaluarse y ser evaluada. El documento base para la implementación de la evaluación externa es el informe de autoevaluación institucional a cargo de la institución universitaria. En dicho informe se exponen las actividades, la organización y el funcionamiento de la institución, así como sus objetivos, políticas y estrategias; presenta un análisis de los procesos y de los resultados obtenidos y también una apreciación sobre su realidad actual teniendo en cuenta su proyecto institucional y su historia. La segunda instancia del proceso de evaluación institucional, la evaluación externa, se realiza con la participación de un comité de pares ajenos a la CONEAU y a la institución evaluada que, junto al equipo técnico de la CONEAU y a los miembros a cargo, realizan la visita a la institución. El comité de pares elabora la agenda de visita donde se define qué actores de la comunidad universitaria evaluada serán entrevistados.

Actualmente, la Resolución de CONEAU № 382/12 es la normativa que rige el procedimiento de evaluación externa: recupera las ideas generales de los documentos antes mencionados y especifica las dimensiones de análisis. De su lectura, se observa que la normativa funciona como una guía para las instituciones evaluadas y también para los pares evaluadores. En este documento queda claramente establecido cuáles son los aspectos institucionales que deben estar comprendidos en el informe de autoevaluación institucional. 


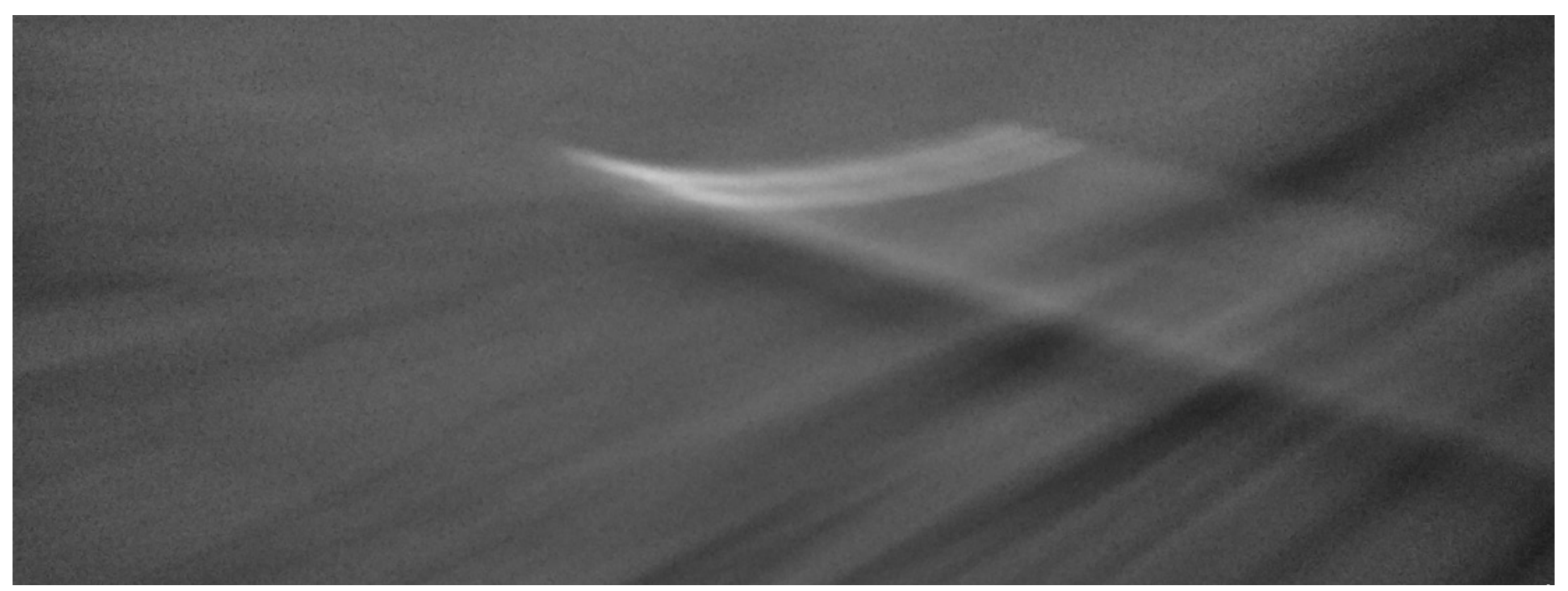

(C) Amanda Merino

En este sentido, refiere a la información mínima pero no excluye la posibilidad de incorporar otra información relevante para la institución que efectúa el proceso evaluatorio y tampoco cerca el análisis que pretenda realizar el comité de pares convocado. Además, de la lectura de esta Resolución es posible identificar que, con relación a la función "extensión", la CONEAU se interesa principalmente por evaluar si la estructura de gestión de la función es apropiada para su desarrollo, si existen políticas explícitas de extensión, producción de tecnología y transferencia afines a la misión y los objetivos institucionales; si esas políticas favorecen la generación de programas de extensión, producción de tecnología y transferencia; si existen políticas de formación de los recursos humanos destinados a esta función; si el presupuesto asignado resulta suficiente así como el equipamiento y los recursos materiales asignados, si existe una política definida de convenios para el desarrollo de las actividades; si la institución cuenta con proyectos que articulen las funciones universitarias (extensión, investigación y docencia) en los que participen alumnos, docentes e investigadores.

El proceso culmina con un informe que recoge la experiencia de la evaluación institucional, el análisis de las funciones evaluadas y las recomendaciones de la CONEAU organizadas por dimensiones:

- Gestión (Gestión y Gobierno).

- Docencia (Gestión Académica).

- Investigación (Investigación, Desarrollo y Creación Artística).

- Extensión (Extensión, Producción de Tecnología y Transferencia).

Es de destacar que todas las decisiones sobre la constitución del comité de pares, así como sobre el informe final de evaluación externa, son adoptadas por el plenario de la CONEAU.

\section{Aspectos metodológicos de la investigación}

Esta investigación se ha elaborado sobre la base de fuentes documentales, por lo que quedan fuera de la misma el análisis de la dinámica institucional que genera la actividad evaluativa y también las opiniones de los actores involucrados.

Se analizaron 29 informes de evaluación institucional elaborados por CONEAU en el período 1999-2016. La selección de los informes fue aleatoria, la muestra no es representativa pero se prestó especial atención a que los informes analizados en conjunto reflejaran las características del sistema universitario argentino en lo que refiere al tipo de gestión y a la ubicación geográfica de las instituciones. A julio de 2016, la CONEAU había realizado 120 evaluaciones externas: 69 correspondientes a instituciones privadas y 51 a estatales. Por ello, resulta relevante considerar que, de los 29 informes, 17 de ellos corresponden a instituciones universitarias de gestión privada (59\%) y 12 a instituciones de gestión pública (41\%). Según la distribución geográfica, el 58 \% de los informes analizados en este trabajo es de instituciones universitarias ubicadas en el CPRES (Consejos Regionales de Planificación de la Educación Superior) Metropolitano, el $20 \%$ en el CPRES COES (Centro-Oeste), el $7 \%$ en el CPRES CES (Centro-Este), otro 7 $\%$ en el CPRES Noreste; el $4 \%$ al CPRES Sur, y el $4 \%$ en el CPRES Noroeste. Además, del total de los informes seleccionados, 26 corresponden a una primera evaluación institucional, 2 a una segunda evaluación y solo 1 a una tercera. En ningún caso se realizó un seguimiento de una misma universidad con varias evaluaciones institucionales a lo largo del tiempo.

De estos documentos se tuvo en cuenta especialmente el apartado de las recomendaciones elaboradas por la CONEAU. Al interior de este apartado, es posible advertir las recomendaciones para 
cada función. Las recomendaciones sobre la función extensión fueron organizadas en las siguientes subdimensiones: políticas de extensión; proyectos y actividades de extensión; vinculación con el medio (convenios); articulación al interior de la universidad; estructura de organización y bienestar estudiantil (becas) y graduados. ${ }^{2}$

\section{La extensión universitaria según los informes de evaluación externa}

Los 29 informes de evaluación externa cuentan con un total de 585 recomendaciones que, tal como se observa en la Tabla 4, se organizan por función. El 28 \% corresponde a Gestión y gobierno, el $44 \%$ a la función Docencia (Gestión Académica), el $18 \%$ a Investigación, desarrollo y creación artística y el 10 \% a la función Extensión, producción de tecnología y transferencia.

La muestra de análisis de la función "extensión" está conformada por 62 las recomendaciones vinculadas a la extensión universitaria, de las cuales el 57 \% (35) corresponde a instituciones de gestión privada y el $43 \%$ (27) a instituciones de gestión estatal.

Como se observa en la Tabla 5, respecto de la categoría "Política de extensión”, el 82 \% (9) de las recomendaciones CONEAU las realiza a instituciones privadas y el $18 \%$ (2) a estatales. Sobre las recomendaciones en torno a los proyectos y actividades de extensión, el 64 \% (9) corresponde a universidades privadas y el $36 \%$ a instituciones de gestión pública. En cuanto a las recomendaciones atinentes a la vinculación con el medio (convenios), el $83 \%$ (5) se efectúa a instituciones privadas y el $17 \%$ (1) a instituciones públicas. Sobre las recomendaciones que refieren a la articulación del área de extensión al interior de la universidad, el $45 \%$ (56) se realiza a universidades privadas y el $55 \%$ (6) a universidades públicas. Con relación a las recomendaciones sobre estructura de organización, el 58 \% (7) corresponde al sector privado y el $42 \%$ (5) al sector público. Por último, y a diferencia de todas las categorías anteriores, las recomendaciones sobre bienestar estudiantil, becas y graduados, refieren en su totalidad (8) a universidades públicas.

Del análisis de las recomendaciones sobre la política de extensión surge que estas son mucho más frecuentes en las universidades de gestión privada que en el caso de las universidades públicas. La mitad de las instituciones de gestión privada analizadas (9 de 17) tiene un desarrollo incipiente de la función extensión universitaria y todavía no cuenta con una política integral para el desarrollo de la misma. Cabe destacar que esta situación se observa en los primeros informes de evaluación externa realizados por la CONEAU

Tabla 4: Recomendaciones seleccionadas según dimensiones y tipo de gestión universitaria

\begin{tabular}{|c|c|c|c|c|c|c|}
\hline \multirow[t]{3}{*}{ Dimensiones } & \multicolumn{4}{|c|}{ Tipo de gestión } & \multirow[t]{3}{*}{ Total } & \multirow[t]{3}{*}{$\%$} \\
\hline & \multicolumn{2}{|c|}{ Gestión Pública } & \multicolumn{2}{|c|}{ Gestión Privada } & & \\
\hline & $\%$ & Cantidad & $\%$ & Cantidad & & \\
\hline Gestión & 39 & 63 & 61 & 100 & 163 & 28 \\
\hline Docencia & 50 & 129 & 50 & 127 & 256 & 44 \\
\hline Investigación & 31 & 32 & 69 & 72 & 104 & 18 \\
\hline Extensión & 43,5 & 27 & 56,5 & 35 & 62 & 10 \\
\hline Totales & 43 & 251 & 57 & 334 & 585 & 100 \\
\hline
\end{tabular}

Fuente: elaboración propia.

Tabla 5: Recomendaciones sobre extensión según subdimensión y tipo de gestión universitaria

\begin{tabular}{|c|c|c|c|c|c|}
\hline \multirow{3}{*}{$\begin{array}{l}\text { Extensión: } \\
\text { subdimensiones }\end{array}$} & \multicolumn{4}{|c|}{ Tipo de gestión } & \multirow[t]{3}{*}{ Total } \\
\hline & \multicolumn{2}{|c|}{ Gestión Pública } & \multicolumn{2}{|c|}{ Gestión Privada } & \\
\hline & $\%$ & Cantidad & $\%$ & Cantidad & \\
\hline Políticas de extensión & 82 & 9 & 18 & 2 & 11 \\
\hline $\begin{array}{l}\text { Proyectos y actividades } \\
\text { de extensión }\end{array}$ & 64 & 9 & 36 & 5 & 14 \\
\hline $\begin{array}{l}\text { Vinculación con } \\
\text { el medio }\end{array}$ & 83 & 5 & 17 & 1 & 6 \\
\hline $\begin{array}{l}\text { Articulación al interior } \\
\text { de la universidad }\end{array}$ & 45 & 5 & 55 & 6 & 11 \\
\hline $\begin{array}{l}\text { Estructura de } \\
\text { organización }\end{array}$ & 58 & 7 & 42 & 5 & 12 \\
\hline $\begin{array}{l}\text { Bienestar estudiantil, } \\
\text { becas y graduados }\end{array}$ & 0 & 0 & 100 & 8 & 8 \\
\hline Totales & 56,5 & 35 & 43,5 & 27 & 62 \\
\hline
\end{tabular}

Fuente: elaboración propia.

dría decirse que bienestar estudiantil, becas y graduados corresponden a la función docencia.

de extensión desarrollaba en su universidad o equivalente. No significa una posición teórica sobre las dimensiones que corresponden o no al desarrollo de la función. A modo de ejemplo, pocategorías de la función (subdimen nes) se realizó en virtud de los datos obtenidos en los informes y de las áreas de trabajo que cada secretaría 
y también en los últimos. Al respecto, la CONEAU explicita la necesidad de que estas universidades privadas definan el concepto institucional de extensión y formulen una política integral para la gestión del área.

A modo de ejemplo, se destacan las siguientes recomendaciones: "Definir e instituir una política integral para la extensión y la prestación de servicios a la comunidad por parte de la Universidad a los fines de lograr una planificada vinculación intra e interinstitucional”. (Recomendación realizada a una institución universitaria privada, CPRES Metropolitano, 2014).

"Desarrollar una política de extensión y crear un área de responsabilidad que impulse actividades de extensión en el ámbito del instituto destinadas a la promoción de la salud en aquellos sectores que más necesitan de este tipo de trabajo. Especialmente, este tipo de actividades tendría un alto impacto social en la sede del interior así como en sectores más vulnerables fuera de la CABA". (Recomendación realizada a una institución universitaria privada, CPRES Metropolitano, 2000).

Con relación a los proyectos y actividades, en una proporción similar la CONEAU recomienda incrementar la cantidad de actividades y sistematizar su registro tanto en las universidades de gestión privada (9 de 17) como públicas (5 de 12). Asimismo, recomienda definir la modalidad y sistematizar la convocatoria, el seguimiento y la evaluación de proyectos. En esta subdimensión, también son recurrentes las recomendaciones que solicitan se fomente la participación de la comunidad universitaria en las actividades de extensión (estudiantes y docentes).

"Fortalecer la iniciativa de formar una base de datos que registre todas las actividades de Extensión Universitaria así como sus alcances y resultados". (Recomendación realizada a una institución universitaria privada, CPRES Metropolitano, 2009).

"Establecer alguna modalidad de gestión, promoción, coordinación, control y evaluación de sus actividades de extensión, de las Facultades y de la Universidad como un todo". (Recomendación realizada a una institución privada, CPRES Nuevo Cuyo, 2000).

Sobre la vinculación con el medio, la CONEAU recomienda incrementar la firma de convenios con organismos públicos e instituciones educativas. En reiteradas ocasiones, los señalamientos apuntan a que las universidades regularicen vinculaciones con instituciones y/o organismos que vienen sosteniendo de manera informal.

"Intentar llevar a cabo actividades de extensión a través de la firma de convenios con Organismos Públicos". (Recomendación realizada a una institución privada, CPRES Metropolitano, 2009).
"Mantener y fortalecer los vínculos actuales que la Universidad mantiene con organismos públicos y privados especialmente a través de convenios firmados con ellos". (Recomendación realizada a una institución privada, CPRES Nuevo Cuyo, 2000).

Sobre la articulación del área de extensión al interior de la universidad, las recomendaciones de CONEAU se orientan en señalar la necesidad de avanzar en la vinculación entre actividades de docencia, investigación y extensión coordinadas institucionalmente. Para lograrlo, la CONEAU propone distintas estrategias, algunas de ellas ligadas al desarrollo o incremento de criterios de valoración de la extensión en la carrera docente y en la formación de los estudiantes.

"Jerarquizar la Función Extensión como función académica sustantiva de la Universidad, incorporando criterios de valoración y evaluación de estas actividades en la carrera docente y/o los concursos, en la formación de estudiantes, en la participación de los graduados y en las funciones de los no docentes". (Recomendación realizada a una institución pública, ubicada en todo el país, 2012).

En el caso de las universidades que prácticamente no han desarrollado la función de extensión universitaria (en su mayoría de gestión privada), las recomendaciones de CONEAU indican la necesidad de conformar una estructura de organización en tanto espacio institucional de gestión de la extensión (regulado y debidamente reglamentado) o bien, en universidades donde el desarrollo de esta función es incipiente, redefinir las funciones del espacio institucional asignado (indicando metas y plazos a corto, mediano y largo plazo) y evitar su superposición.

"Conformar una instancia de gestión de la Extensión

Universitaria que tenga atribuciones en lo referente a la orientación, registro, coordinación y supervisión de todas las actividades que encuadran en esta función, de manera de optimizar los recursos disponibles". (Recomendación realizada a una institución privada, CPRES Metropolitano, 2009).

Las recomendaciones sobre bienestar estudiantil, becas y graduados son señalamientos específicos que surgen de la situación particular de cada una de las instituciones evaluadas: avanzar en el desarrollo del comedor universitario y nuevas residencias, planificar actividades deportivas en las sedes, incrementar las difusiones de becas estatales o desarrollar estrategias de contención e integración de graduados, entre otras.

"Teniendo en cuenta la proyección de futuros egresados para los próximos años resulta necesario pensar estrategias de integración y contención de graduados a partir de espacios de trabajo y de espacios de vinculación". (Recomendación realizada a una institución pública, CPRES Metropolitano, 2009). 


\section{6}

Del análisis de los informes de evaluación externa elaborados por la CONEAU surge que las recomendaciones referidas a la función extensión son menores con relación a las efectuadas sobre otras funciones

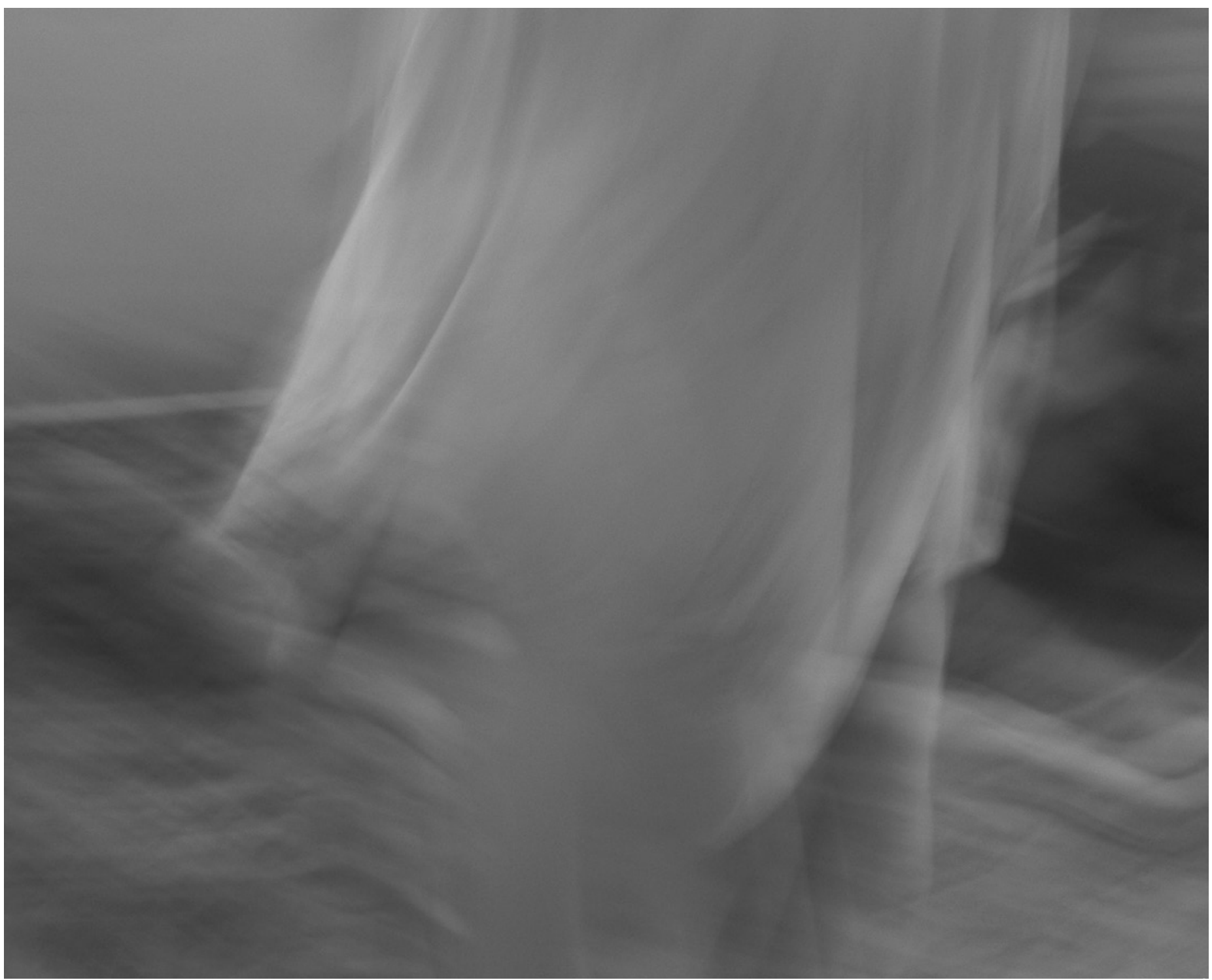


"Avanzar en los proyectos de comedor universitario y nuevas residencias". (Recomendación realizada a una institución pública, CPRES Centro, 2010).

Del análisis de los informes de evaluación externa elaborados por la CONEAU que formaron parte de la muestra utilizada en esta investigación (especialmente los capítulos referidos a la dimensión bajo análisis, el apartado de las conclusiones y las recomendaciones finales) surge que las recomendaciones referidas a la función extensión son menores con relación a las efectuadas sobre otras funciones.

Por el contenido de las recomendaciones se puede inferir que: a) La extensión universitaria ocupa todavía un lugar relegado dentro de la jerarquía universitaria si se la compara con el desarrollo de las funciones de docencia e investigación.

b) Las instituciones privadas han desarrollado escasamente esta función.

c) Las universidades públicas han desarrollado la extensión en mayor magnitud en los últimos años debido la asignación de partidas presupuestarias específicas otorgadas por el Ministerio de Educación de la Nación y otros organismos nacionales.

Asimismo, del análisis de cada subdimensión en particular, se observa que las recomendaciones varían según el grado desarrollo de la función en cada universidad.

En el caso de las universidades privadas, que poseen un exiguo desarrollo de la función, las recomendaciones se orientan a dos aspectos:

a) Definir el concepto institucional de extensión. La CONEAU identifica que no todas las universidades cuentan con una definición institucional que exprese el posicionamiento de la universidad sobre los alcances de esta función y, en esos casos, insta a las instituciones a generar espacios de reflexión que permitan arribar a un posicionamiento institucional.

b) Elaborar una política para el área. Se recomienda la formulación de una política integral debido a que muchas universidades carecen de instrumentos que definan las funciones y las reglamentaciones del área específica, a la vez que tampoco cuentan con una estructura organizativa que garantice su desarrollo o que sea acorde a las definiciones institucionales. Tampoco poseen, en determinadas situaciones, con presupuesto específico ni con personal calificado, técnico o administrativo.

En el caso de las universidades privadas que sí han desarrollado la extensión, las recomendaciones se diversifican y se dirigen a los siguientes aspectos:

a) Incrementar la cantidad de actividades de extensión. Aquí, con el objetivo de promover el desarrollo de la función, la CONEAU propone la diversificación de las actividades que se ofrecen, debido a que muchas de ellas son de carácter cultural y/o deportivo, y también un aumento en la cantidad.

b) Sistematizar el registro de las actividades desarrolladas a fin de medir la participación de docentes, investigadores y alumnos y el impacto que tuvieron en la comunidad. El objetivo principal es mejorar la planificación de las mismas y optimizar los recursos disponibles.

c) Definir la modalidad de las convocatorias, el seguimiento y la evaluación de los proyectos presentados.

d) Aumentar la firma de convenios con organismos públicos e instituciones educativas. Debido a la falta de formalidad y al escaso de desarrollo del área de algunas universidades, CONEAU identifica que la firma de convenios permite ampliar los ámbitos de desarrollo de las actividades de extensión por fuera de los límites de cada institución.

e) Articular las actividades de extensión con las de investigación y docencia.

f) En casos específicos, crear Unidades de Vinculación Tecnológicas para realizar acciones de transferencia tecnológica y acceder, de esta manera, a diversas fuentes de financiamiento tanto públicas como privadas, especialmente en aquellas universidades que no cuentan con este tipo de estructura y manifiestan falta de recursos para desarrollar el área.

Con relación a las instituciones públicas, las recomendaciones de la CONEAU hacen referencia a dos aspectos centrales:

a) Mejorar las políticas de bienestar estudiantil que emergen de la situación actual de cada universidad. La CONEAU recomienda avanzar por un lado en el desarrollo y la mejora de la infraestructura (comedor universitario, residencias estudiantiles) y, por el otro, en el incremento de la difusión de becas estatales, la generación de programas específicos de bienestar y el desarrollo de estrategias de vinculación, seguimiento y formación de graduados. Estas recomendaciones se dan en aquellas universidades específicas que consideran a la política de bienestar estudiantil y al seguimiento de graduados como parte de su política de extensión. b) Desarrollar actividades de extensión que permitan la articulación con otras funciones al interior de la universidad. Al igual que en las universidades privadas, CONEAU observa que las actividades de docencia, investigación y extensión habitualmente se desarrollan de manera aislada y es más notoria la articulación entre las dos primeras. La situación de las bajas dedicaciones horarias de los docentes atenta contra la posibilidad de que se desarrollen tareas de investigación y extensión universitaria ligadas a las actividades docentes. A esto se suma el nulo o bajo reconocimiento que la extensión tiene en los concursos docentes, su omisión en los reglamentos de carrera docente y la ausencia de curricularización de la extensión en los planes de estudio que promueva la participación estudiantil. 
66

La CONEAU identifica que no todas

las universidades cuentan con una

definición institucional que exprese el

posicionamiento de la universidad sobre

los alcances de esta función

\section{Conclusiones}

En el presente trabajo se tuvo en cuenta el recorrido que la extensión universitaria tuvo en nuestro país desde su origen de modo de comprender mejor el presente de esta función sustancial de la universidad argentina. Si bien la extensión fue históricamente relegada en el quehacer universitario, a la luz de los documentos analizados se destaca la mayor relevancia que ha adquirido en los últimos años. Esto se observa principalmente en el incremento en la financiación estatal que hubo a través de los distintos programas que se implementaron en la última década: Programa de Voluntariado Universitario; Programa Nacional de Becas Universitarias; Programa Nacional de Becas Bicentenario; Programa Nacional de Becas de Grado TIC; Programa Universidad, Diseño y Desarrollo Productivo; Programa "Universidad y Trabajo Argentino", entre otros.

No obstante, en lo que refiere a lo recolectado a partir de las evaluaciones externas de la CONEAU, es de considerar la menor importancia que esta le otorga en sus recomendaciones en comparación con la que se le asigna a otras funciones universitarias: solo el $10 \%$ corresponde a la extensión universitaria. Esta disparidad responde al hecho de que algunas instituciones no han desarrollado esta función al momento de ser evaluadas institucionalmente o lo han hecho de manera muy incipiente, motivo por el cual las recomendaciones de CONEAU se redujeron a instarlas a que estuvieran a poner en marcha la extensión universitaria de acuerdo con su proyecto institucional. A modo de cierre, es posible considerar que la extensión universitaria no ha tenido un desarrollo homogéneo en el sistema universitario argentino. Las variadas formas en que la extensión ha sido atendida refleja, una vez más, la heterogeneidad de las instituciones que integran el sistema. El rol de la CONEAU, a través de los procesos de evaluación institucional, ha sido evaluar el cumplimiento de las funciones universitarias e instar a las instituciones a definirlas y desarrollarlas cuando su cumplimiento fuera incipiente o nulo y realizar recomendaciones de mejora más concretas en el caso de que las instituciones hayan puesto en marcha una política institucional al respecto. Con la definición de respetar la singularidad de los distintos proyectos institucionales, la CONEAU ha evaluado la función extensión universitaria junto a otra premisa que el organismo sostiene a través del tiempo e independientemente del tipo de gestión: la extensión es una función universitaria que no puede ser omitida. Entendida, en términos generales, como la relación que la institución desarrolla con la comunidad, esta debe estar contenida en el proyecto institucional, debe tener asignada una estructura orgánica que permita su desarrollo, debe promover la participación de la comunidad universitaria y articularse con las actividades de investigación y docencia. Corresponde a las universidades definir el modo en que se desarrollen estos aspectos, sus particularidades y alcances. 


\section{Referencias bibliográficas}

Argumedo, M. L. et al. (2002). Las estrategias de formación de sujetos en los proyectos de extensión universitaria en la UNLP. En Krotsch, P. (Org.) y Prati, M. (Ed.), La Universidad Cautiva. Legados, marcas y horizontes. La Plata: Ediciones Al Margen.

Bianco, C. (2005). Tensión y extensión universitaria. El modo en que la Facultad de Ciencias Jurídicas y Sociales instituye sus lazos con la comunidad. En Actas del Sexto Congreso Nacional de Sociología Jurídica "El Derecho en la Sociedad Actual". Universidad de Buenos Aires.

Brusilovsky, S. (1998). Recuperando una experiencia de Democratización Institucional y social: La extensión universitaria en la universidad de Buenos Aires (1956-1966). Revista del Instituto de Investigaciones en Ciencias de la Educación, VII(12). Buenos Aires.

Cano Menoni, J. A. (2014). La extensión universitaria en la transformación de la universidad latinoamericana del siglo XXI: disputas y desafíos. Buenos Aires: CLACSO

Carlevaro, P. (1986). Extensión universitaria. Cuadernos de Politica Universitaria. Montevideo: Asociación Social y Cultural de Estudiantes de la Enseñanza Pública. Casanova, H. (2012). El gobierno de la Universidad en España. La Coruña: Netbiblos. CEPAL (2014). Invertir para transformar. La juventud como protagonista del desarrollo. Santiago de Chile: Naciones Unidas.

CEPAL y UNFPA (2012). Invertir en juventud. Informe Regional de Población en América Latina y el Caribe 2011. Santiago de Chile: Naciones Unidas.

Chiroleau, A. (2006). Políticas de Educación Superior en Argentina y Brasil de los '90 y sus continuidades. SAAP-Publicación de Ciencia y Política de la Sociedad Argentina de Análisis Político, 2(3), 563-590.

CONEAU (1997). Lineamientos para la evaluación institucional. Buenos Aires. (2011). Avances de Gestión desde la Evaluación Institucional. Disponible en: http://www.coneau.gov.ar/archivos/publicaciones/documentos/AvancesdeGestion.pdf
(2012). La CONEAU y el Sistema Universitario Argentino. Disponible en: http://www.coneau.gov.ar/archivos/publicaciones/documentos/La_CONEAU_y_ el_sistema_universitario_argentino.pdf

Espinoza, J. e Isa, L. (2012). La práctica de Extensión como herramienta de Formación Universitaria Integral. ExT: Revista de Extensión de la UNC, 4(2), s/p. Disponible en: https://revistas.unc.edu.ar/index.php/ext/article/view/1034/1847 (30 de mayo de 2017).

Isa, L. (2012). Orígenes y sentido de la extensión universitaria. ExT: Revista de Extensión de la UNC, 4(2), s/p.

Herrera Albrieu, M. L. (2012). Una mirada sobre la extensión universitaria en argentina. Colombia: UNAL.

Krotsch, P.; Camou, A. y Prati, M. (2007). Evaluando la evaluación. Políticas universitarias, instituciones y actores en Argentina y América Latina. Buenos Aires: Prometeo.

Marquís, C. (2014). Dos décadas de evaluación universitaria. En Atario et al., Evaluación y Acreditación Universitaria: actores y políticas en perspectiva (pp. 15-60). Buenos Aires: Universidad de Palermo.

Pozo, C. (2012). Nuevos desafíos en un escenario de emergencia social: la extensión universitaria y la otredad. Extensión en red. Revista electrónica sobre extensión universitaria, (3), s/p. Facultad de Periodismo y Comunicación Social, UNLP. Disponible en: http://perio.unlp.edu.ar/ojs/index.php/extensionenred/article/view/1248 (consultado 30/05/2017).

Taborda, P. (2010). La formación académica del estudiante extensionista desde el diseño curricular. Revista electrónica sobre extensión universitaria, 1. Facultad de Periodismo y Comunicación Social, UNLP. 1-6 Disponible en: http://perio.unlp. edu.ar/ojs/index.php/extensionenred/article/view/47/22 (consultado 30/05/2017).

Tünnermann, C. (2000). El nuevo concepto de extensión universitaria y difusión cultural y su relación con las políticas de desarrollo cultural en América Latina. Anuario de Estudios Centroamericanos, (4), 93-126.

\section{Evaluaciones externas consultadas}

Universidad Nacional de Tucumán.

Instituto de Enseñanza Superior del Ejército (IESE) (Primera y segunda evaluación externa).

Instituto Universitario de Ciencias de la Salud - Barceló.

Universidad de San Andrés

Universidad de Mendoza.

Universidad Favaloro.

Instituto Universitario Aeronáutico.

Universidad Cuenca del Plata.

Universidad Nacional de Lanús.

Universidad Nacional de La Pampa.

Universidad ISALUD.

Instituto Universitario Gastón Dachary.

Universidad Nacional de la Matanza.

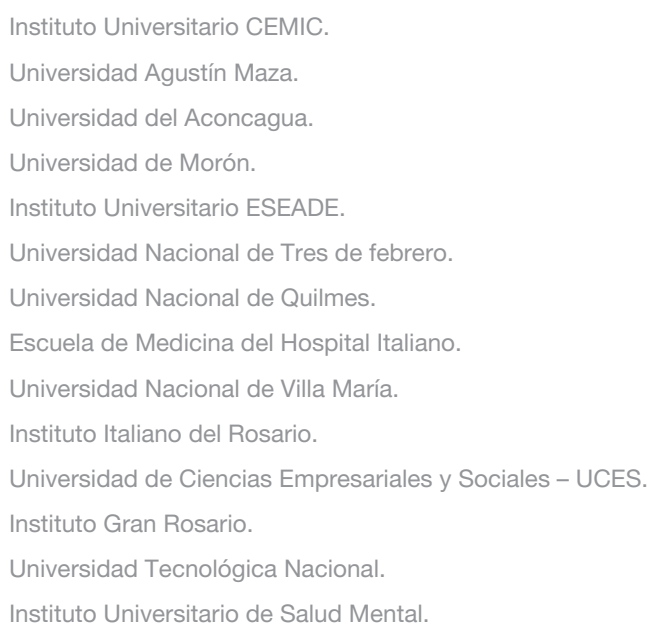

